\title{
COSTO-UTILIDAD DEL TRASPLANTE RENAL FRENTE A LA HEMODIÁLISIS EN EL TRATAMIENTO DE LA INSUFICIENCIA RENAL CRÓNICA TERMINAL EN UN HOSPITAL PERUANO
}

\author{
Ricardo E. Loza-Concha ${ }^{1,2, a}$, Antonio M. Quispe ${ }^{3, b}$
}

\begin{abstract}
RESUMEN
Objetivos. Determinar y comparar las razones de costo-utilidad de los trasplantes renales con donante cadavérico (TRDC) practicados en el Hospital Nacional Guillermo Almenara Irigoyen los años 2000-2001, frente a hemodiálisis (HD), cinco años después del inicio del tratamiento. Material y métodos. Se realizó un estudio de costo utilidad, estudiando a todos los pacientes continuadores cinco años post TRDC, a quienes se asignó como controles, dos pacientes con cinco años de HD pareados por edad, sexo y tiempo de enfermedad. Se evaluaron los costos de cada procedimiento, los años de vida ajustados a la calidad (AVAC) utilizando el cuestionario SF-36v2 ${ }^{\mathrm{TM}}$ y se calcularon las razones de costo utilidad (CU) y costo utilidad incremental (CUI). Resultados. Se realizaron 58 TRDC los años 2000-2001. Cinco años después, 17 (29\%) pacientes fallecieron y 27 (47\%) continuaron con su tratamiento post-TRDC. Treinta y uno (53\%) TRDC fracasaron; $26 \%$ por rechazo al trasplante, $55 \%$ por complicación y $19 \%$ por tratamiento irregular. Los puntajes SF-36v2 ${ }^{\mathrm{TM}}$ promedio obtenidos por los TRDC y HD fueron $95 \pm 13$ y $87 \pm 18$ puntos respectivamente. En ambos años, los AVAC obtenidos por los TRDC y HD fueron de 251 y 229 puntos respectivamente; las razones de CU para los TRDC y los HD fueron de USD 11984 y USD 9243, y la razón de CUI fue de USD 40 669. Conclusiones. Los TRDC realizados los años 2000-2001, cinco años después resultaron menos costo útiles que las HD; y los TRDC realizados el 2000 tuvieron una menor razón de costo utilidad incremental que los efectuados el 2001, probablemente por su mayor razón de tratamientos irregulares.
\end{abstract}

Palabras clave: Trasplante renal; Hemodiálisis; Costo utilidad; Años ajustados a calidad de vida; Calidad de vida; Perú. (fuente: Decs BIREME).

\section{COST UTILITY OF RENAL TRANSPLANT VS. HEMODIALYSIS IN THE TREATMENT OF END STAGE CHRONIC KIDNEY FAILURE IN A PERUVIAN HOSPITAL}

\begin{abstract}
Objectives. To assess and compare the cost utility of the cadaveric donor renal transplant (CDRT) at the Hospital Nacional Guillermo Almenara Irigoyen between 2000 to 2001, against haemodialysis (HD), 5 years after treatment initiation. Materials and Methods. A cost utility study design was used, which evaluated every patient continuing treatment after 5 years of having the CDRT done, and 2 controls consisting in patients having received HD for 5 years, matched by age, sex and disease duration. The costs of each procedure and their Quality-adjusted life years (QALY's) were evaluated using the questionnaire of quality of life SF-36v $2^{\mathrm{TM}}$, finally calculating the cost utility (CU) and incremental cost utility (ICU) ratios. Results. Fifty-eight CDRT were performed between 2000-1. Five years later, 17 (29\%) patients died and only $27(47 \%)$ continued the treatment after CDRT. Out of the 31 patients $(53 \%)$ having treatment failures, $26 \%$ rejected the transplant, $55 \%$ presented a complication and $19 \%$ were irregular. The mean SF-36v2 ${ }^{\mathrm{TM}}$ scores obtained by the CDRT and HD patients were $95 \pm 12$ and $87 \pm 18$ points, respectively. The QALYs obtained by the CDRT and HD groups were 251 and 229 points, respectively; the CU ratios for the CDRT and HD were USD 11,984 and USD 9,243; and the ICU ratio for the period was USD 40,669. Conclusions. CDRTs performed during the years 2000-1 at the HNGAI, were 5 years later surprisingly less cost effective than the HD and CDRT's performed at year 2000 had a lower incremental cost utility ratio that those performed the 2001 , probably because of the highest rate of irregular treatment.
\end{abstract}

Keywords: Renal replacement therapy; Hemodialysis; Cost utility; Quality adjusted life year; Quality of life; Peru (source: MeSH NLM).

\footnotetext{
Servicio de Nefrología, Hospital Nacional Guillermo Almenara Irigoyen - EsSalud. Lima, Perú.

Facultad de Medicina, Universidad Nacional Mayor de San Marcos. Lima, Perú.

U.S. Naval Medical Research Unit Six (NAMRU-6). Lima, Perú.

Médico, Nefrólogo, Magíster en Gestión de la Salud; b Médico, especialista en Estadística en Investigación, Magíster en Epidemiología.
}

$$
\text { Recibido: 06-12-10 Aprobado: 15-06-11 }
$$




\section{INTRODUCCIÓN}

Los trasplantes renales constituyen un problema de salud pública tanto en el Perú como en el mundo ${ }^{(1,2)}$. Con un incremento en el número de pacientes con insuficiencia renal crónica (IRC) e IRC terminal (IRCT). Aún en países como Inglaterra y Estados Unidos, los casos de IRC e IRCT prácticamente se han duplicado en los últimos diez años, creciendo a un ritmo de 5-8 \% al año ${ }^{(3,4)}$. A pesar de los intensos esfuerzos en promover los trasplantes renales y la donación de órganos, el número de donantes y la frecuencia con la que se realizan los trasplantes renales es escasa ${ }^{(5)}$. Debido a ello, no son pocos los investigadores que han advertido que si esto continúa, se podría amenazar gravemente a los sistemas nacionales de salud ${ }^{(6,7)}$.

En Perú, donde la mayoría de personas no cuenta con un seguro y menos del $0,1 \%$ podría financiarse un trasplante renal ${ }^{(8)}$, la única forma de recibir un tratamiento apropiado es a través del seguro social (EsSalud) y los seguros privados, siendo el primero el que más trasplantes renales registra hasta la fecha. A nivel nacional, se estima que en los últimos diez años, en EsSalud, más de 5000 pacientes han recibido tratamiento por IRCT. De estos, poco menos del $1 \%$ han recibido un trasplante renal, el $15 \%$ fallecieron y el $74 \%$ restante reciben diálisis renal, ya sea hemodiálisis $(83 \%)$ o diálisis peritoneal $(17 \%)^{(9)}$. Es decir, la mayoría de las familias se encuentra desprotegida y si uno de sus integrantes padece de IRCT, lo más probable es que no reciba un trasplante renal como tratamiento ${ }^{(10)}$.

Ante este escenario urge la necesidad de generar información que permita a nuestras autoridades tomar las mejores decisiones sobre cómo afrontar este problema y diseñar intervenciones que nos permitan disminuir los efectos de la falta de equidad, eficiencia y calidad en la prestación de servicios en salud en países como el nuestro ${ }^{(11)}$. En el caso específico del manejo de los pacientes con IRCT, la decisión a tomar es sumamente compleja, ¿qué se puede hacer?, ¿priorizar los programas de trasplante de órganos?, ¿priorizar los programas de diálisis renal?, ¿priorizar las estrategias de prevención primaria? Si bien en la literatura médica se presentan una gran cantidad de estudios que intentan responder estas preguntas, solo unos pocos se han realizado en países en países en vías de desarrollo ${ }^{(12-15)}$ y ninguno con resultados extrapolables a nuestra realidad.

Por ello, el objetivo del presente estudio es determinar si los trasplantes renales de donante cadavérico (TRDC) representan una alternativa terapéutica más costo-útil que la hemodiálisis, cinco años postrasplante; utilizando como población de estudio a los pacientes que recibieron un TRDC en el Hospital Nacional Guillermo Almenara Irigoyen (HNGAI) en 2000 y 2001.

\section{MATERIALES Y MÉTODOS}

\section{DISEÑO DE ESTUDIO}

Se realizó un estudio de costo-utilidad, en el que se calculó la razón de costo-utilidad y la razón de costo-utilidad incremental del manejo de los pacientes con IRCT del HNGAI que recibieron un TRDC tomando como controles a los pacientes con IRCT del HNGAI tratados con HD.

\section{POBLACIÓN Y MUESTRA}

La población de estudio estuvo formada por todos los pacientes con IRCT que recibieron un TRDC en el HNGAl entre enero de 2000 a diciembre de 2001, se asignó a cada paciente, dos controles elegidos entre los pacientes con IRCT que ingresaron al programa de hemodiálisis durante el mismo periodo, pareados según edad, sexo y tiempo de enfermedad. Según las estadísticas del departamento de Nefrología, durante el periodo de estudio en el HNGAI, 58 pacientes recibieron un TRDC. Los criterios usados para su selección como receptores de un TRDC fueron: edad (12-55 años), histocompatibilidad (HLA), estado clínico, ausencia de infecciones agudas o crónicas, estado de nutrición y el consenso de los médicos asistentes del Departamento de Nefrología del HNGAl. Como tratamiento coadyuvante todos recibieron anticuerpos policlonales (Timoglobulina $\AA$ ), metilprednisolona (Solumedrol $($ ), micofenolato mofetil (CellCept ${ }^{\circledR}$ ) y ciclosporina $($ Neoral () ).

En una primera etapa del estudio, se realizó un piloto, aplicando el cuestionario SF-36v2 ${ }^{\mathrm{TM}}$ a quince pacientes sometidos a TRDC y quince sometidos a HD. Con ello se calculó que la diferencia a estimar ente los puntajes SF$36 \mathrm{v} 2^{T M}$ era de siete puntos. Utilizando este dato como referencia, se calculó que para detectar una diferencia de siete puntos SF-36v2 ${ }^{T M}$, con un nivel de confianza del $95 \%$ y un poder de estudio del $80 \%$, se requería de un mínimo de 19 TRCD y 38 HD. Basado en estos estimados y buscando maximizar el poder de nuestro estudio, se decidió enrolar a todos los TRDC continuadores que aceptaran dar su consentimiento y participar en el estudio, por lo que la muestra estuvo compuesta por 27 TRDC y $54 \mathrm{HD}$.

\section{RECOLECCIÓN DE DATOS}

Previo consentimiento informado, se aplicó a todos los TRDC y HD la versión española del Cuestionario de Salud SF-36v2 ${ }^{\mathrm{TM}}$ validada por Alonso et al ${ }^{\left({ }^{(16)}\right.}$. El mismo cuestionario fue aplicado a los casos durante sus controles ambulatorios y a los controles durante su sesión de hemodiálisis, al cumplirse los cinco años del seguimiento y solo a los sobrevivientes. Adicionalmente, se 
recolectó la información clínico-epidemiológica (sexo, edad, año de operación, fracasos, abandonos, complicaciones y tipo de complicaciones) de cada sujeto, revisando sus historias clínicas.

Para el cálculo de los costos promedio de cada intervención se recurrió a la unidad de costos del HNGAl quienes proveyeron los costos detallados tanto por TRDC como por HD. Durante este proceso se tomó especial cuidado en considerar que los costos de cada procedimiento debían ser ajustados cada año, que la cantidad de meses en tratamiento variaba según la adherencia del paciente al tratamiento y que si un paciente presentaba una falla del TRDC por cualquier causa, este volvía a terapia con hemodiálisis. En el caso de los pacientes que recibieron un TRDC se consideraron los costos de intervención por concepto del donante (sistema PROCURA, evaluación prequirúrgica, gastos por mantenimiento y gastos directos del intraoperatorio), gastos de intervención por concepto del receptor (gastos de selección, evaluación y preparación del receptor, gastos del intraoperatorio y gastos del postoperatorio hasta el alta del paciente) y los costos por depreciación de los equipos. Para identificar los gastos del seguimiento se consideraron los gastos por consulta externa, fármacos inmunosupresores, gastos de hospitalización y gastos por servicios generales. En el caso de los pacientes sometidos a hemodiálisis se consideraron los costos por sesión de hemodiálisis, consulta externa, fármacos, pruebas de laboratorio y de imágenes, gastos de hospitalización y gastos por servicios generales. En cada caso se realizaron entrevistas con expertos clínicos para identificar insumos y funciones de producción no descritos en el mencionado protocolo, particularmente en lo referente al seguimiento de los pacientes. El modelo utilizado para esta evaluación económica fue el modelo económico propuesto por Arredondo et al (12). En todos los casos la guía de base la constituyeron los protocolos de estudios y procedimientos en el manejo de intervenciones para la IRCT, así como la historia natural de la enfermedad de acuerdo con cada intervención y, sobre todo, el informe detallado provisto por la unidad de contabilidad y costos del hospital.

El coeficiente costo-utilidad se calculó a través de la relación entre el indicador del costo total de cada intervención y el coeficiente de calidad de vida obtenido cinco años después de haber recibido el TR; la razón de costo-utilidad incremental, se calculó tomando como referencia el costo total de cada intervención y la ganancia de utilidad de acuerdo al SF-36v2 ${ }^{T M}$.

\section{ANÁLISIS DE DATOS}

Para describir las características de la población, se utilizaron para las variables numéricas la media, desviación estándar, valores máximo y mínimo, previa comprobación de la distribución normal con el test de Shapiro-Wilk; para las variables categóricas se utilizó su frecuencia y porcentajes.

Para la determinación de los costos, se utilizó como período de referencia monetaria la primera quincena de noviembre de 2006, con un tipo de cambio de S/ 3,25 nuevos soles por cada dólar americano (USD 1).

Para la determinación de la utilidad, primero se calcularon los puntajes del cuestionario de calidad de vida SF36v2 ${ }^{\text {TM }}$ utilizando el SF-36v2 ${ }^{\text {TM }}$ Health Survey Scoring Demonstration (http://www.sf-36.org/demos/SF-36v2. $\mathrm{html}$ ), y luego este puntaje fue transformado a una escala del 0 al 1. Para el cálculo de los años de vida ajustados a la calidad (AVAC) de cada grupo, se multiplicó la utilidad promedio por el número de meses expresado en años que permanecieron intervenidos los pacientes y la cantidad de pacientes intervenidos.

Para la obtención de la razón de costo-utilidad (CU) se procedió a dividir el costo total del procedimiento entre Ios AVAC obtenidos para cada tratamiento. Para la obtención de la razón costo-utilidad incremental (CUI) se procedió a dividir la diferencia de los costos totales entre la diferencia de medias de ambos procedimientos. Para la comparación de costos se usó la prueba de t de Student pareada.

El análisis fue realizado utilizando el programa estadístico STATA versión 11.0 (Stata Corp LP, College Station, Texas).

\section{ASPECTOS ÉTICOS}

La participación de los encuestados fue voluntaria y anónima, otorgando cada uno su consentimiento informado. El estudio fue revisado y aprobado por el Comité Institucional de Ética del Hospital Nacional Guillermo Almenara Irigoyen (Carta 277-CI-OCID-GM-RAA-EsSalud-2004/HNGAl). Posteriormente fue revisado y aprobado como proyecto de tesis para optar por el título de magíster en salud pública por el comité científico de la Universidad Nacional Mayor de San Marcos (UNMSM).

\section{RESULTADOS}

Entre enero de 2000 y diciembre de 2001, un total de 58 pacientes con IRCT recibieron un TRDC, 27 el 2000 y $31(53,5 \%)$ el 2001 , de los cuales el $59 \%$ eran varones, como se ve en la Tabla 1. La edad promedio de los pacientes trasplantados fue de $40 \pm 15$ años (rango: 12-71 años). Cinco años postrasplante renal, el $47 \%$ 
Tabla 1. Características generales de los pacientes sometidos a TRDC. HNGAI: 2000-2001

\begin{tabular}{|c|c|c|c|}
\hline Características & $\begin{array}{c}\text { Año } 2000 \\
N(\%)\end{array}$ & $\begin{array}{c}\text { Año } 2001 \\
N(\%)\end{array}$ & $\begin{array}{l}\text { Total } \\
\mathrm{N}(\%)\end{array}$ \\
\hline Pacientes con TRDC & $27(46,6)$ & $31(53,5)$ & $58(100,0)$ \\
\hline Hombres & $15(55,5)$ & $19(61,2)$ & $34(58,6)$ \\
\hline Edad (Media \pm DE años) & $38,1 \pm 16,7$ & $41,1 \pm 13,4$ & $39,8 \pm 15,0$ \\
\hline \multicolumn{4}{|l|}{ Evolución a los cinco años } \\
\hline Continuadores & $15(55,5)$ & $12(44.5)$ & $27(46,5)$ \\
\hline Fracasos & $12(44,5)$ & $19(61,2)$ & $31(53,4)$ \\
\hline Rechazo & $3(25,0)$ & $5(26,3)$ & $8(25,8)$ \\
\hline Complicaciones & $7(58,3)$ & $10(52,6)$ & $17(54,8)$ \\
\hline Infecciones & $4(57,1)$ & $5(50,0)$ & $9(52,9)$ \\
\hline Tumorales & $2(28,6)$ & $5(50,0)$ & $7(41,2)$ \\
\hline Postoperatorias & $1(14,3)$ & $0(0,0)$ & $1(05,9)$ \\
\hline Tratamiento Irregular & $2(16,7)$ & $4(21,1)$ & $6(19,4)$ \\
\hline \multicolumn{4}{|l|}{ Sobrevida a los cinco años } \\
\hline Vivos & $20(74,1)$ & $21(67,7)$ & $41(70,7)$ \\
\hline Fallecidos & $7(25,9)$ & $10(32,3)$ & $17(29,3)$ \\
\hline Rechazo & $1(14,3)$ & $0(0,0)$ & $1(5,9)$ \\
\hline Complicaciones & $6(85,7)$ & $10(100,0)$ & $16(94,1)$ \\
\hline \multicolumn{4}{|c|}{ Tasas cinco años post TRDC } \\
\hline Tasa de rechazos (\%) & 11,1 & 16,1 & 13,8 \\
\hline Tasa de sobrevida (\%) & 74,1 & 67,7 & 70,7 \\
\hline Tasa de abandono (\%) & 7,4 & 12,9 & 10,3 \\
\hline
\end{tabular}

TRDC, Trasplante renal de donante cadavérico; DE, Desviación estándar

de los pacientes trasplantados continuaron con éxito su tratamiento. Del total de fracasos, el $55 \%$ fueron por complicaciones. De estos, el $53 \%$ se debió a complicaciones infecciosas. A los cinco años, del total de pacientes trasplantados, el $10 \%$ abandonaron el seguimiento y el $29 \%$ falleció. De los 17 pacientes que fallecieron, 16 lo hicieron como consecuencia de alguna complicación postrasplantes y solo uno de ellos por un rechazo al trasplante propiamente dicho, 14 meses después de haber reiniciado su tratamiento con HD.

El costo promedio de cada TRDC y su tratamiento continuo por cinco años asciende a USD 57 431. Tomando este costo como un $100 \%$, el costo atribuible a cada TRDC per se (USD 21 799) se calculó que constituye el $38 \%$ de este costo, como se aprecia en la Tabla 2.

Entre los años 2000 y 2005 cada sesión de HD costó en promedio entre USD 42,7 y USD 52,3. Dado que un paciente recibe en promedio tres sesiones por semana y 156 sesiones al año, el costo mensual promedio del tratamiento con HD varió en entre USD 546 y USD 679 al mes, mientras que el costo anual promedio varió entre USD 6553 y USD 8152, como se ve en la Tabla 3.

Los puntajes SF-36v2 ${ }^{T M}$ obtenidos por los pacientes que recibieron un TRDC son mayores que aquellos que
Tabla 2. Análisis de costos de los pacientes sometidos a TRDC. HNGAI: 2000-2001.

\begin{tabular}{|c|c|c|c|}
\hline \multirow{2}{*}{ Descripción } & \multicolumn{2}{|c|}{ Costo promedio } & \multirow{2}{*}{$\%$} \\
\hline & $\mathrm{S} /$. & USD $\ddagger$ & \\
\hline \multicolumn{4}{|l|}{ Trasplante } \\
\hline \multicolumn{4}{|l|}{ Donante } \\
\hline PROCURA† & 2048 & 630 & 1,1 \\
\hline Evaluación & 1576 & 484 & 0,8 \\
\hline Mantenimiento & 3846 & 1183 & 2,1 \\
\hline Intraoperatorio & 9434 & 2902 & 5,1 \\
\hline Sub total & 16905 & 5201 & 9,1 \\
\hline Receptor & & & 0,0 \\
\hline Selección & 819 & 252 & 0,4 \\
\hline Evaluación & 6092 & 1874 & 3,3 \\
\hline Preparación & 6960 & 2141 & 3,7 \\
\hline Intraoperatorio & 6948 & 2137 & 3,7 \\
\hline Postoperatorio & 30075 & 9253 & 16,1 \\
\hline Sub total & 50895 & 15660 & 27,3 \\
\hline Depreciación de equipos & 3048 & 938 & 1,6 \\
\hline TOTAL & 70849 & 21799 & 38,0 \\
\hline \multicolumn{4}{|l|}{ Seguimiento } \\
\hline \multicolumn{4}{|l|}{ Seguimiento 1 er. Año } \\
\hline Consulta externa & 617 & 190 & 0,3 \\
\hline Fármacos ( $\mathrm{T}, \mathrm{MP}, \mathrm{MF}$ y $\mathrm{C})$ & 21851 & 6723 & 11,7 \\
\hline Hospitalización & 2899 & 892 & 1,6 \\
\hline Servicios generales & 65 & 20 & 0,0 \\
\hline Subtotal & 25434 & 7825 & 13,6 \\
\hline Seguimiento $2 .^{\text {do }}$ año & 24070 & 7406 & 12,9 \\
\hline Seguimiento 3 er $^{\text {er }}$ ão & 23891 & 7351 & 12,8 \\
\hline Seguimiento $4 .^{\text {to }}$ año & 21748 & 6691 & 11,7 \\
\hline Seguimiento $5 .^{\text {to }}$ año & 20659 & 6356 & 11,1 \\
\hline TOTAL & 115803 & 35631 & 62,0 \\
\hline TOTAL GENERAL & 186652 & 57431 & 100,0 \\
\hline
\end{tabular}

$\ddagger$ Tipo de cambio: USD 1 =S/. 3,25; † PROCURA, Programa vigente hasta el año 2005 encargado de procurar los cadáveres e identificar a los posibles donantes candidatos para Transplante renal de donante cadavérico (TRDC); T, Timoglobulina ${ }^{\circledR}$; MP, Metilprednisolona (Solumedrol $囚) ;$ MF, Micofenolato Mofetil (CellCept $\left.{ }^{\circledR}\right)$; C, Ciclosporina (Neoral ${ }^{\circledR}$ ) Fuente: Unidad de Contabilidad y Costos - EsSalud, octubre de 2006.

Tabla 3. Costos de los pacientes sometidos a HD. HNGAI: 2000-2001

\begin{tabular}{cccc}
\hline Año & $\begin{array}{c}\text { Costo/Sesión } \\
\text { S/. (USD })\end{array}$ & $\begin{array}{c}\text { Costo/año } \\
\text { S/. (USD })\end{array}$ & $\begin{array}{c}\text { Costo/mes } \\
\text { S/. (USD }{ }^{\ddagger}\end{array}$ \\
\hline 2000 & $136,5(42,0)$ & $21298(6553)$ & $1774(546)$ \\
\hline 2001 & $138,6(42,6)$ & $21323(6654)$ & $1802(554)$ \\
\hline 2002 & $139,2(42,8)$ & $21716(6682)$ & $1809(556)$ \\
\hline 2003 & $166,0(51,1)$ & $25905(7970)$ & $2158(664)$ \\
2004 & $169,8(52,2)$ & $26495(8152)$ & $2207(679)$ \\
\hline 2005 & $157,1(48,3)$ & $24509(7541)$ & $2042(628)$ \\
\hline
\end{tabular}

‡ Tipo de cambio: USD 1 =S/. 3,25.

Fuente: Unidad de Contabilidad y Costos - EsSalud, octubre de 2006. 
Tabla 4. Calidad de vida según el SF-36v2 ${ }^{T M}$ de los pacientes sometidos a TRDC en los años 2000 y 2001, y de sus controles en tratamiento con HD pareados por edad, sexo y tiempo de enfermedad. HNGAl, octubre de 2006.

\begin{tabular}{cccc}
\hline $\begin{array}{c}\text { Calidad de } \\
\text { Vida }\end{array}$ & $\begin{array}{c}\text { TRDC } \\
\text { (Media } \pm \text { DE) }\end{array}$ & $\begin{array}{c}\text { HD } \\
\text { (Media } \pm \text { DE) }\end{array}$ & \multicolumn{1}{c}{$\boldsymbol{p}$} \\
\hline Salud Física & $41,5 \pm 7,5$ & $40,7 \pm 9,6$ & 0,69 \\
\hline Función Física & $45,2 \pm 6,8$ & $40,0 \pm 11,0$ & $0,03^{*}$ \\
\hline Rol Físico & $40,9 \pm 12,5$ & $39,3 \pm 14,7$ & 0,62 \\
\hline Dolor Corporal & $45,3 \pm 11,0$ & $44,8 \pm 12,8$ & 0,88 \\
\hline Salud General & $43,1 \pm 8,6$ & $41,7 \pm 7,0$ & 0,44 \\
Salud Mental & $53,7 \pm 9,6$ & $46,3 \pm 12,2$ & $0,01^{*}$ \\
Vitalidad & $53,9 \pm 11,7$ & $48,0 \pm 12,8$ & $0,05^{*}$ \\
\hline Función Social & $49,1 \pm 9,8$ & $39,9 \pm 12,3$ & $0,001^{*}$ \\
\hline Rol Emocional & $46,8 \pm 11,9$ & $39,3 \pm 15,2$ & $0,03^{*}$ \\
\hline Salud Mental & $53,4 \pm 9,5$ & $49,6 \pm 12,1$ & 0,16 \\
Puntaje Total & $95,3 \pm 12,1$ & $86,9 \pm 17,7$ & $0,03^{*}$ \\
2000 & $96,1 \pm 11,0$ & $85,5 \pm 16,8$ & $0,03^{*}$ \\
2001 & $94,2 \pm 13,7$ & $88,9 \pm 18,9$ & $0,39^{*}$ \\
\hline
\end{tabular}

DE, Desviación estándar; $p$, valor de significancia; ${ }^{\ddagger}$ prueba de chi $^{2} ;{ }^{*}$, valor de $p$ estadísticamente significativo $(p<0.05)$

recibieron solo $\operatorname{HD}(95,3 \pm 12,1$ frente a $87,0 \pm 17,7$ puntos), siendo esta diferencia significativa ( $p<0,01)$; a su vez, de acuerdo con las ocho dimensiones, respecto a la salud física, solo función física mostró un diferencia significativa entre ambos grupos, mientras que rol físico, dolor corporal y salud general no; respecto a la salud mental, vitalidad, función social y rol emocional, mostraron diferencias significativas, mientras que salud mental no (Tabla 4).

Considerando los costos por unidad contable que se muestran en la Tabla 5, se estimó que el costo total de los TRDC realizados los años 2000 fueron de USD 1344364 y el costo total de los controles en HD de USD 972 349; mientras que el costo total de los TRDC realizados los años 2001 fue de USD 1654500 y el costo total de los controles en HD fue de USD 1116401.

Se encontró que la razón de costo-utilidad en el caso de los pacientes que recibieron un TRDC el 2000 (USD 11397 ) resultó inferior al de los pacientes que recibieron un TRDC el 2001 (USD 12 460), mientras que la razón de costo-utilidad entre los pacientes que iniciaron HD el 2000 (USD 9 269) resultó superior a la encontrada el 2001 (USD 8917). Producto de ello, los TRDC realizados el 2000 y 2001 resultaron ser menos costo-útiles (USD 11 984) que las HD (USD 9243). Finalmente, se pudo calcular que la razón de costoutilidad incremental, para los TRDC practicados el 2000 (USD 28 489) resultó ser inferior a la calculada para los TRDC practicados el 2001 (USD 70 980).
Tabla 5. Costos acumulados de la población de estudio, HNGAI.

\begin{tabular}{|c|c|c|c|}
\hline Concepto & $\begin{array}{l}\text { Costo por } \\
\text { unidad USD/ }\end{array}$ & $\begin{array}{l}\text { Número } \\
\text { contable* }\end{array}$ & $\begin{array}{l}\text { Costo } \\
\text { USD/ }\end{array}$ \\
\hline TRDC & 21799,8 & 58 & 1264387,8 \\
\hline \multicolumn{4}{|l|}{$\begin{array}{l}\text { Meses post } \\
\text { TRDC }\end{array}$} \\
\hline $1^{\text {er }}$ año & 652,2 & 674 & 439555,8 \\
\hline 2. do año & 617,2 & 550 & 339454,5 \\
\hline 3. er año & 612,6 & 405 & 248103,0 \\
\hline $4 .^{\text {to }}$ año & 557,6 & 356 & 198519,8 \\
\hline $5 .^{\text {to }}$ año & 529,7 & 332 & 175867,0 \\
\hline \multicolumn{4}{|l|}{ Meses HD } \\
\hline Año 2000 & 546,1 & 0 & 0,0 \\
\hline Año 2001 & 554,5 & 33 & 18299,2 \\
\hline Año 2002 & 556,8 & 97 & 54013,5 \\
\hline Año 2003 & 664,2 & 148 & 98307,5 \\
\hline Año 2004 & 679,4 & 156 & 105980,2 \\
\hline Año 2005 & 628,4 & 108 & 67871,5 \\
\hline Costo Total & & & 3010359,9 \\
\hline \multicolumn{4}{|l|}{ Hemodiálisis } \\
\hline \multicolumn{4}{|l|}{ Meses HD } \\
\hline Año 2000 & 546,1 & 324 & 176942,9 \\
\hline Año 2001 & 554,5 & 696 & 385945,9 \\
\hline Año 2002 & 556,8 & 696 & 387560,6 \\
\hline Año 2003 & 664,2 & 696 & 462311,0 \\
\hline Año 2004 & 679,4 & 696 & 472834,6 \\
\hline Año 2005 & 628,4 & 372 & 233779,7 \\
\hline Costo Total & & & 2119374,7 \\
\hline
\end{tabular}

TRDC, Trasplante Renal de Donante Cadavérico; HD, Hemodiálisis.

* Número de meses/paciente por procedimiento año.

Tabla 6. Coeficientes de Costo Utilidad para cada intervención y coeficientes de Costo Utilidad Incremental para cada año, HNGAl: 2000-2001.

\begin{tabular}{|c|c|c|c|c|}
\hline Año & $\begin{array}{l}\text { Costo } \\
\text { US\$/ }\end{array}$ & $\begin{array}{c}\text { Puntaje } \\
\text { SF-36v2 }^{T M}\end{array}$ & AVAC & $\begin{array}{l}\text { CU } \\
\text { USD }\end{array}$ \\
\hline \multicolumn{5}{|l|}{ Año 2000} \\
\hline TRDC & 1344364 & 96,1 & 118,0 & 11397 \\
\hline $\mathrm{HD}$ & 972349 & 85,5 & 104,9 & 9269 \\
\hline CUI & 372014 & 10,6 & 13,1 & 28489 \\
\hline \multicolumn{5}{|l|}{ Año 2001} \\
\hline TRDC & 1654500 & 94,2 & 132,8 & 12460 \\
\hline $\mathrm{HD}$ & 1116401 & 88,9 & 125,2 & 8917 \\
\hline CUI & 538098 & 5,4 & 7,6 & 70980 \\
\hline \multicolumn{5}{|c|}{ Años 2000 y 2001} \\
\hline TRDC & 3010359 & 95,3 & 251,2 & 11984 \\
\hline $\mathrm{HD}$ & 2119374 & 87,0 & 229,3 & 9243 \\
\hline CUI & 890985 & 8,3 & 21,9 & 40669 \\
\hline
\end{tabular}

AVAC, Años de vida ajustados a la calidad; CU, Razón de Costo utilidad (Costo/AVAC); TRDC, Trasplante Renal de Donante Cadavérico; HD, Hemodiálisis; CUI, Razón de Costo Utilidad Incremental ([Costo TRDC - Costo HD]/ [AVAC TRDC - AVAC HD]) 


\section{DISCUSIÓN}

En el presente estudio, contrario a lo esperado, se concluye que los TRDC realizados en el HNGAI en 2000 y 2001, cinco años después, no son más costo-útiles que los HD. Más aun, se encontró que los TRDC realizados el 2001 resultaron más costosos y menos útiles que los realizados el 2000. Si bien esto puede deberse a la principal limitación del presente trabajo, que es no incluir un análisis de costos indirectos, nuestros datos parecen indicar que la principal causa del alto costo y poca utilidad alcanzados por los TRDC practicados en el HNGAI se debe al elevado costo que resulta realizar un TRDC en nuestro medio y las altas tasas de fracasos.

De acuerdo con la literatura, el hecho de no incluir el análisis de costos indirectos no invalida los análisis econométricos ${ }^{(17)}$. De acuerdo con la revisión sistemática publicada por Clark et al., no solo no existe una metodología estándar para estimar los costos indirectos, sino que la gran diversidad de métodos utilizados para calcularlos casi invalidan su comparación (18). Por otro lado, algunas de las principales amenazas contra el balance costo utilidad resultan ser: i) el alto costo de los TRDC propiamente dichos (aproximadamente USD 22000 cada uno); ii) los altos costos generados por los pacientes durante el tratamiento de soporte (USD 36000 a los cinco años); iii) la relativa poca ganancia en términos de $\operatorname{AVAC}(7,6-13,1)$; iv) las altas tasas de rechazos (cerca al $25 \%$ ) y sus complicaciones; y v) la baja adherencia de los receptores de un TRDC al tratamiento inmunosupresor, registrada en nuestro estudio como tratamientos irregulares (cerca a un $20 \%$ ).

Cabe precisar que los TRDC realizados durante el 2000 y 2001 en el HNGAI, son relativamente menos costosos que los TRDC realizados en países como Estados Unidos ${ }^{(19)}$ y Bélgica ${ }^{(20)}$, por ejemplo. Sin embargo, el por qué no resultan ser costo-útiles pueda deberse al encarecimiento de los TRDC conforme pasan los años, debido a las altas tasas de fracasos, complicaciones y tratamientos irregulares. Cada paciente con TRDC que fracasa o se complica, en teoría, debería pasar a HD, generando un doble gasto a la institución; sin embargo, la mayoría fallece; el por qué requiere otro estudio, pero para cuestiones de discusión de nuestros resultados, estos también generan un impacto directo en el incremento de los costos por TRDC.

La mejora de la calidad de vida observada en los pacientes trasplantados con respecto a los hemodializados según algunos estudios, se puede explicar por la mayor movilidad del paciente, el menor estrés al que se ven sometidos, su mayor grado de independencia, así como a la recuperación de su capacidad productiva ${ }^{(21,22)}$. Gracias al trasplante, los pacientes tienen mayores posibilidades de realizar un trabajo remunerado puesto que no interrumpen sus actividades cotidianas(21). Así mismo, los pacientes trasplantados sufren menos estrés ya que informan estar menos deprimidos, preocupados, ansiosos o insatisfechos que los pacientes en diálisis ${ }^{(23)}$. Ogutmen et al., por ejemplo, comparando una serie de 302 casos de TRDC frente a 64 casos de HD, 207 en diálisis peritoneal y 278 controles normales, describieron que la calidad de vida alcanzada por los receptores de un TRDC era marcadamente superior a la de pacientes que seguían en HD, sin embargo, aún distaba de la calidad de vida un adulto sano ${ }^{(24)}$.

Un punto que llamó nuestra atención fueron los altos puntajes SF-36v2 ${ }^{\mathrm{TM}}$ alcanzados por los pacientes en tratamiento con hemodiálisis. Si bien estos no son tan diferentes a los descritos por Martins et al. ${ }^{(25)}$, que informó que la calidad de vida en 125 pacientes en tratamiento con HD (28 \pm 24 meses en tratamiento), utilizando el puntaje SF-36 ${ }^{\mathrm{TM}}$ (en portugués), llegan a alcanzar puntajes altos de calidad de vida (total 105,9 $\pm 5,7$ puntos). Nuestros valores son más altos que los informados por Valisieva et al. ${ }^{(26)}$,que luego de estudiar 1047 pacientes en tratamiento con HD, también utilizando el SF-36 ${ }^{\mathrm{TM}}$, informaron puntajes bajos de calidad de vida (total $81,1 \pm 10,5$ puntos), que son significativamente bajos comparados con controles sanos, particularmente en los puntajes de salud física, excepto en la escala de salud mental donde sorpresivamente se obtuvo una media mayor que la de los pacientes sanos ${ }^{(26)}$. De la misma manera, Guerini-Rocco et al., luego de analizar una serie de 47 pacientes en tratamiento con HD informaron que estos también pueden alcanzar SF-36 ${ }^{\mathrm{TM}}$ muy bajos (total, 50,1 $\pm 22,6$ ) comparados con controles sanos (total, $91,9 \pm 23,4)^{(1)}$.

Un asunto aparte es la adherencia al tratamiento postrasplante que es un problema a nivel mundial (27). En nuestro caso se hace evidente si notamos que los trasplantes renales realizados el 2000 tuvieron una menor razón de costo-utilidad incremental que los trasplantes renales realizados durante el 2001, que 27 de los 58 pacientes sometidos a TRDC pueden ser considerados éxitos cinco años postrasplante; que los 27 TRDC realizados el 2000 costaron en total USD 1344364 cinco años después, y los 31 TRDC realizados el 2001, costaron USD 1654 500. Por otro lado, los costos de las HD practicadas en el HNGAl fueron incluso más baratas que el costo promedio a nivel mundial, alcanzando costos atribuibles a HD practicadas en condiciones ideales (hemodiálisis domiciliaria nocturna, hemodiálisis no hospitalaria o satélite y otras variantes de la hemodiálisis intrahospitalaria más económicas) ${ }^{(28,29)}$. Esto se debe 
probablemente a que los establecimientos de EsSalud reprocesan los filtros de HD buscando abaratar costos. Por último, si bien el tratamiento con anticuerpos policlonales es más costo-efectivo que los tratamientos con las generaciones anteriores de inmunosupresores ${ }^{(30)}$, en nuestro estudio las altas tasas de complicaciones tumorales como infecciosas sugieren lo contrario, lo que debería ser investigado.

De acuerdo con la literatura, la salud mental de los pacientes con IRCT es muy sensible al TR, mejorando significativamente hasta quince años después, donde ya muestra una clara tendencia a la normalidad. Neipp et al., por ejemplo, luego de hacer un seguimiento a un grupo de 139 pacientes 15 años post TRDC, informan que cuatro de las ocho escalas del SF-36v2 $2^{\mathrm{TM}}$ se encontraban en niveles normales, la salud mental entre ellas, (rol físico, función social, rol emocional y salud mental) mientras que el resto (función física, dolor físico, salud general y vitalidad) se encontraban aún disminuidas ${ }^{(2)}$. Sin embargo, en nuestro estudio si bien es cierto existen diferencias claras en los puntajes totales del SF-36v2 ${ }^{\mathrm{TM}}$ entre los receptores de un TRDC comparados con los pacientes que permanecen en HD, no se encontraron mayores diferencias en los puntajes que miden la salud mental.

En general, en términos de eficiencia económica, los TRDC realizados el 2000 tuvieron una mejor razón de costo-utilidad incremental comparados con los efectuados el 2001. Esto probablemente se deba a que la mayor cantidad de pacientes continuadores pertenecen al grupo del 2000 y, pese a que en el 2001 se operaron la mayoría de los pacientes que sobrevivieron, en ese año también se registraron cuatro de los seis casos de abandono, diez de las 17 complicaciones y cinco de los ocho rechazos. Desde una perspectiva económica, cada paciente que abandona la terapia de mantenimiento, eventualmente, se complica y regresa a HD, generando un gasto enorme que no es retribuido en utilidades. Más aún aquellos que se complican y fallecen, generan un costo de oportunidad muy alto puesto que no solo representan una inversión fallida sino que generan un gasto sostenido pues deben recibir HD. La no adherencia a la terapia post TR es una de las principales causas de falla del TR a nivel mundial (27).

Es importante precisar que en el HNGAI, durante el 2000 y 2001 , el programa de TRDC que estuvo vigente (PROCURA) estipulaba que aquellos candidatos a donantes cadavéricos (pacientes con muerte cerebral, mayores de 12 y menores de 55 años, con buena función renal, clínicamente estables y libres de infecciones agudas o crónicas), de acuerdo con su histocompatibilidad debían ser inmediatamente trasplantados a los mejores receptores posibles de la lista de espera. Esto implicaba que de haber más de un candidato primaba entre otros el factor preparación del paciente, es decir, aquel paciente que eventualmente presentaba la mayor disposición y disponibilidad de internamiento era el que se beneficiaba con el trasplante, sin embargo esta respuesta carecía de una evaluación de salud mental exhaustiva.

En función de nuestros resultados, nosotros consideramos que se debe hacer todo lo posible para controlar las altas tasas de abandono del tratamiento registradas por nuestros sujetos de estudio. Estos casos no solo generan un gasto enorme a la institución y no representa utilidades significativas sino que también constituyen una oportunidad perdida de tratar a un paciente que aproveche mejor la oportunidad que se brindó.

En conclusión, en las condiciones en los que los TRDC fueron realizados el 2000 y 2001 en el HNGAI, cinco años después, todavía no son más costo-útiles que los HD. Principalmente por las diferencias en las tasas de abandono y rechazos, los TRDC realizados el 2000 tuvieron una menor razón de costo-utilidad incremental que los TRDC efectuados el 2001.

\section{Contribuciones de autoría}

RELC y AMQ participaron en la concepción y diseño del estudio, RELC lideró la recolectaron los datos, AMQ supervisó el avance del estudio, $A M Q$ realizó el control de calidad de los datos y realizó el análisis de los datos. Ambos autores interpretaron los resultados y participaron en la redacción y aportes críticos del artículo así como en la aprobación de la versión final a publicar.

\section{Fuentes de financiamiento}

Autofinanciado.

\section{Conflictos de interés}

Los autores declaran no tener conflictos de interés en la ejecución y publicación de este artículo.

\section{Descargo de responsabilidad}

Antonio M. Quispe es actualmente un empleado del Gobierno de los EE.UU. Las opiniones y afirmaciones contenidas aquí son propias de los autores y no deben interpretarse como posición oficial o que reflejen la opinión del Departamento de la Marina o del Servicio Naval de los estados Unidos.

\section{REFERENCIAS BIBLIOGRÁFICAS}

1. Guerini Rocco D, Mercieri A, Yavuzer G. Multidimensional health-status assessment of chronic hemodialysis patients: the impact on quality of life. Eura Medicophys. 2006;42(2):113-119. 
2. Neipp M, Karavul B, Jackobs S, Meyer zu Vilsendorf A, Richter N, Becker T, Schwarz A, Klempnauer J. Quality of life in adult transplant recipients more than 15 years after kidney transplantation. Transplantation. 2006;81(12):16401644.

3. USRDS: the United States Renal Data System. American journal of kidney diseases : the official journal of the National Kidney Foundation. 2003;42(6 Suppl 5):1-230.

4. Byrne C, Ford D, Gilg J, Ansell D, Feehally J. UK Renal Registry 12th Annual Report (December 2009): chapter 3: UK ESRD incident rates in 2008: national and centre-specific analyses. Nephron Clin Pract. 2010;115 Suppl 1:c9-39.

5. Lu AD, Carter JT, Weinstein RJ, Stratta RJ, Taylor RJ, Bowers VD, Ratner LE, Chavin KD, Johnson LB, Kuo PC, Cole EH, Dafoe DC, Alfrey EJ. Outcome in recipients of dual kidney transplants: an analysis of the dual registry patients. Transplantation. 2000;69(2):281-285.

6. DuBose TD, Jr. American Society of Nephrology Presidential Address 2006: chronic kidney disease as a public health threat--new strategy for a growing problem. Journal of the American Society of Nephrology : JASN. 2007;18(4):10381045.

7. Knauf F, Aronson PS. ESRD as a window into America's cost crisis in health care. Journal of the American Society of Nephrology: JASN. 2009;20(10):2093-2097.

8. INEI. Encuesta Nacional de Hogares 2003 (ENAHO) http:// www.inei.org.pe (Revisado el 30/10/2006). 2003.

9. Saavedra-López A. Epidemiología de la IRC. X Congreso Internacional de Nefrología - Sociedad Peruana de Nefrología. 14 al 17 Septiembre, Lima Perú. 2006.

10. Guzmán N, Ballenas J, Medina M, Chavarry W, Berrios C, Quiroa L, Seminario M, Calderón F, Ardiles A, Vásquez G, Liendo C, Bardales F, Bravo J, Ramírez E, Castillo R, Espejo J, Castro P, Camacho M, Mendez P, Gómez M, Meneses V. Transplante renal en el Hospital Edgardo Rebagliati Martins. X Congreso Internacional de Nefrología - Sociedad Peruana de Nefrología. 14 al 17 Septiembre, Lima Perú. 2006.

11. Brenzel L. Application of cost-effectivenss analysis to decision-making in the health sector of developing countries. Washington: World Bank. 1993

12. Arredondo A, Rangel R, de icaza E. Costs of intervention for patients with chronic renal disease. Rev Saude Publica. 1998;32:255-261.

13. Gregory MC. Cost-effective dialysis for the developing world. Ethn Dis. 2009;19(1 Suppl 1):S1-65-67.

14. Naidas OD, Chan-Licuanan KR, Velasco VP, Dalay CV, Bayog DV, Rosete-Liquete RM. Cost effectiveness analysis of alternative treatments of end-stage renal disease: Philippine experience. Transplant Proc. 1998;30(7):3116.

15. Sumboonnanonda A, Lumpaopong A, Kingwatanakul P, Tangnararatchakit K, Jiravuttipong A. Pediatric kidney transplantation in Thailand: experience in a developing country. Transplantation proceedings. 2008;40(7):22712273.

16. Alonso J, Prieto L, Anto JM. La versión española del SF36 Health Survey (Cuestionario de Salud SF-36): un instrumento para la medida de los resultados clínicos. Med Clin (Barc). 1995;104:771-776.
17. Erickson KF, Winkelmayer WC. The challenges of costeffectiveness analyses for the clinician. American journal of kidney diseases : the official journal of the National Kidney Foundation. 2010;56(6):1023-5.

18. Clarke KS, Klarenbach S, Vlaicu S, Yang RC, Garg AX. The direct and indirect economic costs incurred by living kidney donors-a systematic review. Nephrol Dial Transplant. 2006;21(7):1952-1960.

19. Chisholm MA, Vollenweider LJ, Mulloy LL, Wynn JJ, Wade WE, DiPiro JT. Cost-benefit analysis of a clinical pharmacist-managed medication assistance program in a renal transplant clinic. Clin Transplant. 2000;14(4 Pt 1):304307.

20. Chaib-Eddour D, Chaib-Eddour H, Malaise J, Mourad M, Squifflet JP. Cost of renal transplant in Belgium. Transplant Proc. 2005;37(6):2819-2820.

21. Niu SF, Li IC. Quality of life of patients having renal replacement therapy. J Adv Nurs. 2005;51(1):15-21.

22. Gokal R. Quality of life in patients undergoing renal replacement therapy. Kidney Int Suppl. 1993;40:S23-27.

23. Lopes A, Frade IC, Teixeira L, Oliveira C, Almeida M, Dias L, Henriques AC. Depression and anxiety in living kidney donation: evaluation of donors and recipients. Transplantation proceedings. 2011;43(1):131-136.

24. Ogutmen B, Yildirim A, Sever MS, Bozfakioglu S, Ataman R, Erek E, Cetin O, Emel A. Health-related quality of life after kidney transplantation in comparison intermittent hemodialysis, peritoneal dialysis, and normal controls. Transplant Proc. 2006;38(2):419-421.

25. Martins MR, Cesarino CB. [Quality of life in chronic kidney failure patients receiving hemodialysis treatment]. Rev Lat Am Enfermagem. 2005;13(5):670-676.

26. Vasilieva IA. Quality of life in chronic hemodialysis patients in Russia. Hemodial Int. 2006;10(3):274-278.

27. Butler JA, Roderick P, Mullee M, Mason JC, Peveler RC. Frequency and impact of nonadherence to immunosuppressants after renal transplantation: a systematic review. Transplantation. 2004;77(5):769-776.

28. Roderick P, Armitage A, Nicholson T, Mehta R, Gerard K, Mullee M, Drey N, Lamping D, Feest T, Greenwood R, Townsend J. A clinical and cost evaluation of hemodialysis in renal satellite units in England and Wales. Am J Kidney Dis. 2004;44(1):121-131.

29. Soroka SD, Kiberd BA, Jacobs $\mathbf{P}$. The marginal cost of satellite versus in-center hemodialysis. Hemodial Int. 2005;9(2):196-201.

30. Lilliu H, Brun-Strang C, Le Pen C, Buchler M, AI Najjar A, Priol G, Reigneau O, Lebranchu Y. Cost-minimization study comparing Simulect vs. Thymoglobulin in renal transplant induction. Clin Transplant. 2004;18(3):247-253.

Correspondencia: Antonio M. Quispe

Dirección: Pedro Conde 340, Oficina 201, Lince, Lima, Perú.

Teléfono: (511) 421-9504 / (511) 614-4141 anexo 215 /

(511) 99672-0197

Correo electrónico: antonio.quispe@med.navy.mil 